\title{
A situação dos ensaios clínicos relacionados às doenças mentais comuns
}

\author{
The situation of clinical trials related to common mental disorders
}

El estado de los ensayos clínicos relacionados con enfermedades mentales comunes

Gisele Aparecida Fófano ${ }^{1}$, Letícia Vieira da Silva ${ }^{1 *}$, Marcio Fernandes dos Reis ${ }^{2}$, Hiara Lopes Pinheiro Teixeira ${ }^{1}$, Guilherme de Andrade Avelar ${ }^{1}$, Fernanda Cyrino de Abreu ${ }^{1}$, Maria Augusta Coutinho de Andrade Oliveira ${ }^{1}$.

\section{RESUMO}

Objetivo: Analisar e revisar sistematicamente a situação dos ensaios clínicos sobre transtornos mentais comuns disponíveis na base de dados ClinicalTrials.gov. Métodos: Estudo ecológico desenvolvido na base de dados pública e gratuita Clincaltrials.gov, que define a situação dos Ensaios Clínicos em Não Recrutados; Recrutando; Inscrições por convite; Ativo, não recrutando; Encerrado; Completo; Completo com resultados; Suspenso; Retirado ou Desconhecido. O site foi acessado através dos descritores: "Ansiedade" e "Depressão". Nenhum projeto foi excluído do estudo. Essa busca foi feita em 2019. Resultados: Dos estudos, $47,76 \%$ foram financiados por indústrias, $41,55 \%$ pelo National Institutes of Health $(\mathrm{NIH})$ e 10,69\% pela Agência Federal dos Estados Unidos. Percebe-se que apenas 18,4\% do total de ensaios clínicos geraram resultados. Além disso, quanto à população estudada, 32,72\% são crianças, $91,27 \%$ são adultos e $57,05 \%$ são idosos. Considerações finais: Torna-se cada vez mais importante pensar nos vieses, não só para os ensaios clínicos em Saúde Mental, mas para todos os outros estudos que venham a ser realizados, valorizando a ética na publicação de resultados positivos e negativos em todas as circunstâncias observadas.

Palavras-chave: Assistência à saúde mental, Ansiedade, Depressão.

\section{ABSTRACT}

Objective: To analyze and review systematically the situation of clinical trials on common mental disorders available in the ClinicalTrials.gov database. Methods: Ecological study developed in the public and free database Clincaltrials.gov, which defines the situation of Clinical Trials in Not yet Recruting; Recruting; Enrolling by invitation; Active, not recruiting; Terminated; Completed; Completed with results; Suspended; Withdraw or Unknown. The site was accessed using the descriptors: "Anxiety" and "Depression". No projects were excluded from the study. This search has been done in 2019. Results: Of the studies, $47.76 \%$ were financed by industries, $41.55 \%$ by the National Institutes of Health $(\mathrm{NIH})$ and $10.69 \%$ by the US Federal Agency. It is noticed that only $18.4 \%$ of the total clinical trials generated results. In addition, as for the study population $32.72 \%$ are children, $91.27 \%$ are adults, and $57.05 \%$ are elderly. Final considerations: It becomes increasingly important to think about biases, not only for clinical trials in Mental Health, but for all other studies that may be carried out, valuing ethics in publishing positive and negative results in all observed circumstances.

Key words: Mental health assistance, Anxiety, Depression.

${ }^{1}$ Centro Universitário Governador Ozanam Coelho (UNIFAGOC), Ubá - MG.

*E-mail: leticiavieiradasilva@gmail.com

${ }^{2}$ Centro Universitário Presidente Antônio Carlos (UNIPAC), Juiz de Fora - MG.

SUBMETIDO EM: 2/2021

ACEITO EM: 2/2021

PUBLICADO EM: 3/2021 


\section{RESUMEN}

Objetivo: Analizar y revisar sistemáticamente la situación de los ensayos clínicos sobre trastornos mentales comunes disponibles en la base de datos ClinicalTrials.gov. Métodos: Estudio ecológico desarrollado en la base de datos pública y gratuita Clincaltrials.gov, que define la situación de los Ensayos Clínicos en Aún No Reclutamiento; Reclutando; Inscribirse por invitación; Activo, no reclutando; Terminado; Terminado; Completado con resultados; Suspendido; Retirar o Desconocido. Se accedió al sitio utilizando los descriptores: "Ansiedad" y "Depresión". No se excluyó ningún proyecto del estudio. Esta investigación se ha realizado en 2019. Resultados: De los estudios, el 47,76\% fueron financiados por industrias, el $41,55 \%$ por los Institutos Nacionales de Salud (NIH) y el 10,69\% por la Agencia Federal de Estados Unidos. Se observa que solo el $18,4 \%$ del total de ensayos clínicos generaron resultados. Además, en cuanto a la población de estudio, el $32,72 \%$ son niños, el $91,27 \%$ son adultos y el $57,05 \%$ son ancianos. Consideraciones finales: Cada vez es más importante pensar en los sesgos, no solo para los ensayos clínicos en Salud Mental, sino para todos los demás estudios que se puedan realizar, valorando la ética en la publicación de resultados positivos y negativos en todas las circunstancias observadas.

Palabras clave: Asistencia en salud mental, Ansiedad, Depresión.

\section{INTRODUÇÃO}

Os transtornos mentais comuns (TMC) são compostos por sintomas não psicóticos que incluem ansiedade, depressão e transtornos somatoformes relacionados ao estresse (MURCHO N, et al., 2016). Porém, para a Organização Mundial da Saúde (OMS), embora o conceito permaneça o mesmo, os transtornos relacionados são apenas ansiedade e depressão (OMS, 2017).

Para essas doenças, os dados da OMS relativos a 2017, indicam que no mundo existem cerca de 44 milhões de pessoas afetadas pela depressão e, em média, 28 milhões de pessoas com ansiedade. Na América Latina, existem 1.271.941.170 indivíduos pela primeira, enquanto existem 1.296.939.210 pessoas afetadas pela segunda causa. Isoladamente, o Brasil é responsável por cerca de $97,48 \%$ do total de depressivos e $97,07 \%$ dos ansiosos no continente latino-americano (GHDx, 2017).

Ainda existem pessoas vivendo com as duas doenças, cuja prevalência ainda não foi estimada (OMS, 2017). Para tentar reduzir estes e outros números, foi lançado em 2013 um plano de ação relacionado com a saúde mental, que duraria até 2020. Este plano contém 4 objetivos básicos, o último dos quais está relacionado com o fortalecimento das evidências e pesquisas científicas relacionadas. à saúde em relação aos transtornos mentais.

Além disso, um dos princípios desse plano é promover a prática baseada em evidências, que envolve a análise de publicações científicas atualizadas (OMS, 2013). Aderindo a esse movimento crescente relacionado ao aumento do conhecimento sobre o tema, destaca-se a integridade científica, que pode ser definida como a adoção de boas práticas em pesquisa, que visam, além de proteger os autores do plágio, evitar a publicação de dados improváveis, como bem como conduta antiética em qualquer momento da pesquisa (PÁDUA GCC e GUILHEM D, 2015; PINTO AC, 2015).

Por todo o seu papel, é amplamente responsável pela formulação das políticas institucionais dos principais sistemas de pesquisa em todo o mundo e também tem desempenhado um papel importante na promoção da confiança pública na ciência produzida por esses sistemas (VASCONCELOS S, et al., 2015).

Principalmente em tempos de pandemia, é importante pensar no futuro da saúde mental, inicialmente porque $\mathrm{o}$ isolamento, a perda de rendimentos e o pânico, podem ser desencadeadores ou agravar os transtornos mentais, no contexto atual espera-se que algumas pessoas tenham insônia, ansiedade ou aumento do consumo de álcool e níveis de drogas. Além disso, os serviços de saúde mental de muitos países foram afetados negativamente pela pandemia, o que pode prever um grande número de pessoas 
desassistidas tanto em relação aos medicamentos quanto à própria assistência institucional (OMS, 2020). Para ter acesso ao panorama geral dos estudos científicos sobre TMC, a fim de projetar as possibilidades de cumprimento ideal do quarto ponto em escopos globais, é possível acessar o registro de ensaios clínicos.

Devido à ausência de estudos desta natureza na literatura, o objetivo deste estudo é analisar através de uma revisão sistemática a situação dos ensaios clínicos sobre transtornos mentais comuns disponíveis na base de dados ClinicalTrials.gov. Com isso, espera-se que a pesquisa em saúde mental seja valorizada além das intervenções clássicas e receba mais incentivos financeiros globais, principalmente após o atual estado de calamidade.

\section{MÉTODOS}

É uma revisão sistemática de literatura desenvolvida utilizando unicamente informações da base de dados pública e gratuita Clincaltrials.gov em 2019, que define a situação dos Ensaios Clínicos e deve ser constantemente atualizado pelos responsáveis pelo projeto. Foram utilizadas na busca os descritores: "Ansiedade" e "Depressão".

Todos os projetos encontrados através das palavras-chave "Ansiedade" ou "Depressão" foram incluídos, mesmo aqueles que não se tratavam exclusivamente de uma destas duas doenças. O critério de exclusão foi não utilizar o descritor em suas palavras-chave mesmo que seu título fosse relacionado a este tema, a compilação final dos escolhidos encontra-se disponível na Figura 1 abaixo. Todos os selecionados tiveram sua situação analisada de acordo com os marcadores disponíveis no site, que são: Não Recrutando; Recrutamento; Inscrições por convite; Ativo, não recrutando; Encerrado; Concluído; Concluído com resultados; Suspenso; Retirado e Desconhecido, que são traduzidos e descritos de forma quantitativa. Para a formação da tabela e cálculos estatísticos simples, foi utilizado o Microsoft Excel 2016.

Figura 1 - Estudos selecionados de acordo com as palavras-chave e critérios de inclusão e exclusão.

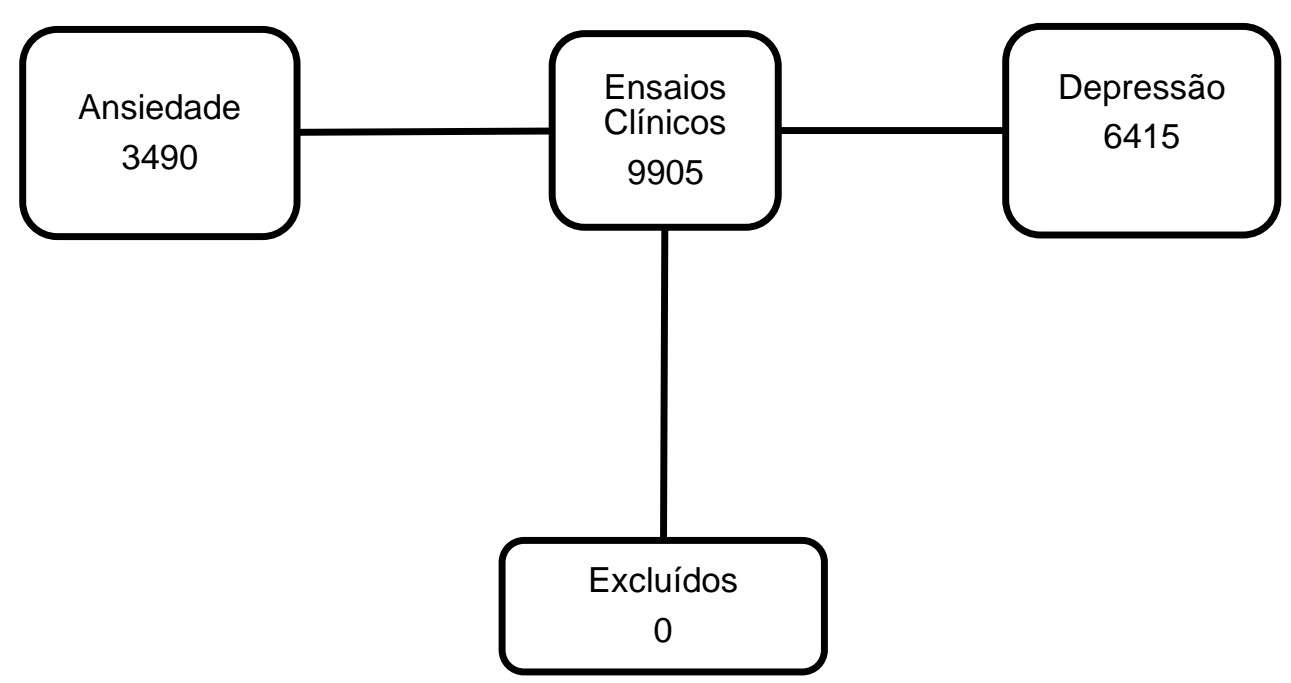

Fonte: Fófano GA, et al, 2021.

\section{RESULTADOS}

Os resultados disponibilizados variam entre diferentes tipos de estudos intervencionistas, podendo estes utilizarem medicações, alterações de hábito de vida, terapias psicológicas ou outras terapias em seus estudos. Independente disso, é importante considerar que eles também são classificados de acordo com seu status, o desconhecido é atribuído a pesquisas cujas datas de conclusão já passaram de 2 anos e seu status 
não foi verificado, enquanto o completo com resultados é aquele que não recruta participantes e já possui seus dados publicados em alguma revista ou na própria base de dados. Uma visão geral dos 9905 Ensaios Clínicos disponíveis sobre depressão e ansiedade são descritos na Tabela 1.

Tabela 1 - Status dos ensaios clínicos para DMCs classificados pela OMS.

\begin{tabular}{|c|c|c|c|}
\hline \multirow{12}{*}{ 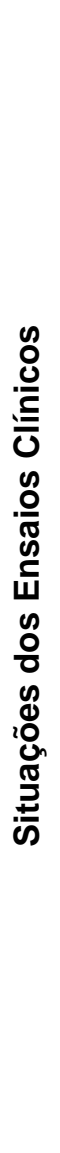 } & Doença & Depressão & Ansiedade \\
\hline & Não Recrutando & 293 & 202 \\
\hline & Recrutando & 1062 & 661 \\
\hline & Recrutando por convite & 85 & 48 \\
\hline & Ativo, não recrutando & 290 & 162 \\
\hline & Terminado & 328 & 138 \\
\hline & Completo & 3627 & 1886 \\
\hline & Completo com resultados & 843 & 319 \\
\hline & Suspenso & 15 & 7 \\
\hline & Retirado & 134 & 69 \\
\hline & Desconhecido & 578 & 316 \\
\hline & Total & 6415 & 3490 \\
\hline
\end{tabular}

Fonte: Fófano GA, et al, 2021; dados extraídos de Clinicaltrials.gov, 2019.

\section{Sobre Financiamento}

Do total de estudos, 3509 tiveram entidades financiadoras, excluindo universidades, organizações e estudos patrocinados individualmente. Do total, $47,76 \%$ foram financiados pelas indústrias, $41,55 \%$ pelos National Institutes of Health (NIH) e 10,69\% pela Agência Federal dos Estados Unidos da América. Ressaltase que os estudos financiados correspondem a 9,44\% dos estudos relacionados aos TMC.

Ao avaliar o destino final do investimento, fica claro que $18,4 \%$ do total de ensaios clínicos geraram resultados. As promovidas pela Agência Federal dos Estados Unidos da América (EUA) foram as que tiveram maior aproveitamento, com $23,15 \%$ das obras convertidas em resultados.

Do total de estudos dessa doença, cerca de $26,79 \%$ foram financiados de alguma forma. Em relação aos estudos realizados pelas indústrias, 1.287 foram direcionados à depressão, correspondendo a 20,06\% dos estudos sobre o tema e $36,68 \%$ dos ensaios financiados. Destes, $26,96 \%$ de todos os testes foram utilizados cientificamente. Porém, ainda existem $5,28 \%$ que podem não gerar dados científicos, enquanto $7,23 \%$ ainda têm esse potencial porque já foram finalizados.

Observa-se também que a Indústria e a Agência Federal dos Estados Unidos possuem aproximadamente o mesmo percentual na relação entre estudos completos e apresentação de resultados, constituindo em média 36,59\% desses resultados. As demais dos ensaios clínicos estão descritas na Tabela 2. 
Tabela 2 - Ensaios clínicos sobre depressão de acordo com a idade.

\begin{tabular}{|c|c|c|c|c|c|c|c|c|}
\hline \multirow{2}{*}{\multicolumn{2}{|c|}{ Variáveis }} & $\mathbf{C}$ & CCR & $\mathbf{T}$ & $\mathbf{R}$ & $\mathbf{S}$ & D & To \\
\hline & & \multicolumn{7}{|c|}{ Depressão } \\
\hline \multirow{3}{*}{ 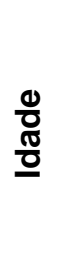 } & Criança (-17 a.i.) & 521 & 81 & 35 & 15 & 1 & 89 & 943 \\
\hline & Adulto (18-64 a.i.) & 3418 & 792 & 310 & 128 & 14 & 544 & 6037 \\
\hline & Idoso (+65 a.i.) & 2711 & 667 & 243 & 99 & 10 & 421 & 4753 \\
\hline \multirow{3}{*}{ 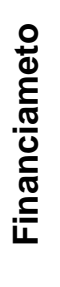 } & Indústria & 965 & 347 & 93 & 19 & 1 & 48 & 1287 \\
\hline & $\begin{array}{l}\text { Instituto Nacional de } \\
\text { Saúde (NIH) }\end{array}$ & 725 & 191 & 46 & 14 & 2 & 27 & 1020 \\
\hline & $\begin{array}{l}\text { Agência Federal dos } \\
\text { Estados Unidos }\end{array}$ & 172 & 64 & 5 & 10 & 0 & 15 & 267 \\
\hline \multicolumn{9}{|c|}{ Ansiedade } \\
\hline \multirow{3}{*}{ 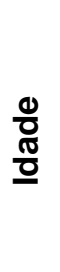 } & Criança (-17 a.i.) & 1227 & 212 & 89 & 41 & 4 & 209 & 2298 \\
\hline & Adulto (18-64 a.i.) & 1621 & 274 & 115 & 61 & 6 & 274 & 2998 \\
\hline & Idoso (+65 a.i.) & 476 & 67 & 34 & 21 & 1 & 88 & 898 \\
\hline \multirow{3}{*}{ 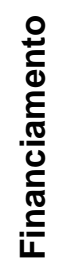 } & Indústria & 277 & 73 & 37 & 3 & 0 & 21 & 389 \\
\hline & $\begin{array}{c}\text { Instituição Nacional de } \\
\text { Saúde (NIH) }\end{array}$ & 310 & 74 & 14 & 8 & 0 & 9 & 438 \\
\hline & $\begin{array}{l}\text { Agência Federal dos } \\
\text { Estados Unidos }\end{array}$ & 66 & 25 & 0 & 1 & 1 & 10 & 108 \\
\hline
\end{tabular}

Legenda: C- Completo; CCR- Completo com resultados; T- Concluído; R- Retirado; S- suspenso; DDesconhecido; To- Total de ensaios clínicos; a.i.: anos de idade.

Fonte: Fófano GA, et al, 2021; dados extraídos de Clinicaltrials.gov, 2019.

Dentre todos os estudos de TMC, nota-se que $32,72 \%$ contém crianças, $91,27 \%$ contém adultos e $57,05 \%$ contém idosos. Já os relacionados à depressão, a maioria envolve apenas a população adulta, chegundo a $94,11 \%$ do total de estudos relacionados a essa doença, sendo seguida pelos estudos relacionados a idosos, correspondendo a $74,10 \%$ do total de estudos.

Observa-se que o site não permite separar estudos que aceitam apenas uma faixa etária daqueles que aceitam mais de uma, portanto, não é possível ter acesso aos números absolutos de cada faixa etária isoladamente. Porém, percebe-se que a maioria dos estudos atinge crianças e adultos, com $65,85 \%$ e $85,90 \%$ respectivamente, quando comparados aos estudos gerais.

\section{Quanto ao sexo}

Novamente, o site oferece apenas opções em que homens e mulheres são considerados em conjunto, a partir desses dados é possível inferir que 635 estudos sobre depressão foram direcionados apenas para mulheres, enquanto aproximadamente 251 estudos relacionados à ansiedade foram dedicados a este público, não sendo possível analisar quantos estudos foram dedicados apenas aos homens, mas pode-se perceber que o resultado foi inferior aos direcionados às mulheres (Tabela 3). 
Tabela 3 - Distribuição dos estudos relacionados ao sexo dos participantes.

\begin{tabular}{ccc}
\hline Transtornos Mentais & \multicolumn{2}{c}{ Sexo } \\
\cline { 2 - 3 } Comuns & Feminino & Masculino \\
\hline Depressão & 6326 & 5691 \\
Ansiedade & 3415 & 3164
\end{tabular}

Fonte: Fófano GA, et al, 2021; dados extraídos de Clinicaltrials.gov, 2019.

\section{DISCUSSÃO}

Em 2016, 61\% de todos os artigos publicados oriundos de pesquisas de saúde mental continham pelo menos um dos três tópicos: doenças cognitivas e neurodegenerativas; depressão, ansiedade e transtornos de personalidade; transtorno de uso e adicção em substâncias (POLLITT A, et al., 2016).

No contexto atual, as pesquisas devem ser estimuladas a serem continuadas, uma vez que, para $89 \%$ dos países incluem a saúde mental e o suporte psicoterapêutico em seu plano de ação contra o coronavírus, entretanto, apenas $17 \%$ dos mesmos possuem fundos financeiros para custear tais atos (WHO, 2020).

Há projeções que indicam que esta pandemia possa desencadear outra: a das doenças mentais, devido ao estresse vivido neste período, relacionado tanto a perdas econômicas, quanto ao adoecimento, às perdas, aos procedimentos necessários para a recuperação e até os métodos utilizados para a própria proteção (MARI JJ e OQUENDO MA, 2020).

Outro fator que propicia o investimento em pesquisa é o grande potencial lucrativo. Para cada dólar gasto em medicina baseada em evidência para depressão e ansiedade, 5 são retornados, assim, a longo prazo, poderia-se observar uma redução no total de 1 trilhão de dólares perdidos anualmente de alguma forma para estas doenças (WHO, 2020).

Percebe-se que o número de estudos relacionados à Depressão é maior do que aqueles relacionados à ansiedade, independente da fonte de financiamento. Talvez isso se deva à maior prevalência da primeira doença no mundo, destacando-se entre os TMC em quase todos os continentes, exceto na região das Américas (OMS, 2017). Este último fato chama a atenção, pois as fontes de financiamento norte-americanas (NIH e Outras Agências Federais dos EUA) trazem um maior percentual de estudos sobre ansiedade.

Por outro lado, nas pesquisas sobre depressão, a existência de outras fontes de financiamento, provindas da preocupação com a elevada prevalência em diversas outras regiões, não tornam este destaque norteamericano tão expressivo. Esses dados contradizem o que foi encontrado por Riveros C, et al (2013), visto que a indústria dominava o financiamento de todas as pesquisas, demonstrando que fortuitamente houve um maior envolvimento de outras instituições patrocinadoras ao longo dos anos.

Ainda quanto a fontes financiadoras das pesquisas em saúde mental, em 2016, dos 10 maiores investidores, 5 eram estadunidenses, e estas eram citadas em $67 \%$ dos artigos produzidos com algum tipo de incentivo financeiro, ficando o $\mathrm{NIH}$ em primeiro lugar em número de artigos produzidos, com $15 \%$. Apesar da maioria das produções científicas serem apoiadas por ações governamentais, pesquisadores em geral reconhecem as instituições sem fins lucrativos e de caridade como as maiores apoiadoras das pesquisas, representando $39 \%$ das instituições reconhecidas pelos mesmos, isso inclui organizações que investem em saúde mental mesmo não tendo este como objetivo principal, a exemplo da British Heart Fundation (POLLITT A, et al., 2016).

Entretanto uma dificuldade que pode culminar em perda de recursos nas pesquisas em saúde mental são quanto a definições explícitas do que está sendo pesquisado, inclusive quanto às ferramentas diagnósticas e os transtornos, se esta for criada poderá permitir a comparação e discussão de portfólios de pesquisa, fato que seria valioso para ajudar os financiadores a descobrir o que outros financiadores estão apoiando e assim contribuir para co-operação financeira internacional, reduzindo as dificuldades de manter os níveis de investimento em uma pesquisa já iniciada permitindo a criação de uma agenda a longo prazo, culminando no incentivo ao término das pesquisas e divulgação de seus resultados (POLLITT A, 2016). 
Quanto aos estudos com resultado, 9,15\% foram estudos sobre ansiedade e $13,14 \%$ dos estudos de depressão. Os estudos de melhor aproveitamento para a ansiedade foram financiados pelas Outras Agências Federais dos Estados Unidos, enquanto para a depressão foi proveniente da Indústria. É compreensível que tanto o governo norte-americano quanto as indústrias, sobretudo as últimas, estejam investindo nestes insumos afinal estes arrecadaram US\$13,755 milhões só em 2016, e a América do Norte foi a líder no consumo de antidepressivos em 2016, e deve continuar até 2023 nesta posição (ALLIED MARKET RESEARCH, 2018). Tendo em vista que certos antidepressivos também atuam como ansiolíticos, isso demontra um duplo ganho financeiro para o mercado farmacológico (HELP GUIDE, 2019).

Neste quesito a integridade científica, questiona-se o conflito de interesses entre a indústria farmacêutica e outras formas de financiamento, uma vez que esses tipos de pesquisas tendem a ter um maior número de resultados favoráveis, portanto, o tipo de financiamento pode contribuir para um viés sobre os resultados fato que requer atenção dos estudiosos da medicina baseada em evidências. Neste ponto, ressalta-se que a integridade científica ainda tem sido pouco debatida nas instituições de ensino que estimulam a o que pode se tornar uma ausência perigosa para o futuro, exigindo incentivo pesquisa (PÁDUA GCC e GUILHEM D, 2015).

Estudos desatualizados, são chamados de invisíveis conhecidos como abandonados, não são novidade no meio acadêmico, sendo considerada como uma forma de levar os profissionais de saúde a conclusões inválidas, tanto benéficas quanto maléficas (FELDMAN AM, 2013). Uma lista publicada por Doshi $P$, et al. (2013) incluía fármacos amplamente utilizados para tratamentos psiquiátricos, quetiapina e paroxetina, e analisava a além da publicação dos resultados, a presença e disponibilidade de dados dos participantes, do ensaio clínico e intercorrências, sendo que para estas informações a maioria dos ensaios omitia estes dados. Este ato impossibilita a verificação da acurácia das informações e outras formas de pesquisa a partir de dados pré-gerados, tanto por outros pesquisadores quanto por revistas (DOSHI P, et al., 2013).

Nesse sentido, os vieses do estudo devem ser considerados e descritos principalmente no momento da publicação dos artigos, uma vez que estando na base de dados, é possível ter acesso à evolução do estudo. E essa é uma parte importante da transparência, que é essencial para garantir a integridade científica (NIH, 2019). No entanto, deve-se questionar o fato de outros estudos, mesmo completos, não apresentarem seus resultados.

A falha no estudo, seja ele terapêutico, comercial ou de segurança, pode ser uma justificativa, visto que apenas $40 \%$ dos resultados negativos são publicados, ressalta-se também que quando o problema é na questão comercial, o dado é ainda menos provável de ser lançado (HWANG TJ, et al., 2016). Fato semelhante pode estar ocorrendo com obras de status desconhecido, uma vez que uma observação negativa pode levar ao abandono do estudo.

Ademais, todos os viéses que permeiam a produção e publicação de artigos relacionados a transtornos mentais, podem ser uma justificativa para que artigos de saúde mental que reconheçam o apoio de instituições de caridade ou fundações e organizações beneficentes tendem a ter um impacto de citação ligeiramente maior do que aqueles que reconhecem outros setores, tornando-os mais dispersáveis e relevantes na literatura (POLLITT A, et al., 2016).

É importante enfatizar que a publicação científica, deve se basear não só na descrição boa e verdadeira dos resultados, mas também na pontuação dos métodos realizados e os interesses devem ser deixados de lado. Deve-se ter cuidado na produção do manuscrito, pois achados incorretos, plágio e retratações podem fazer com que a credibilidade da pesquisa seja perdida, isso é grave, pois afeta o mais alto nível de evidência, que vem da clínica ensaios (LISKAUSKAS S, et al., 2019). O fato de haver mais estudos que incluem mulheres do que aqueles que o fazem com homens, em geral, talvez pelo fato de serem mais diagnosticados com ansiedade e depressão em todas as faixas etárias (OMS, 2017).

Esse perfil de participantes de ensaios clínicos, felizmente, segue uma tendência oposta ao ocorrido há 10 anos, fato que auxilia no melhor entendimento dos efeitos colaterais em todas as populações, reduz 0 vazio informativo, e permite uma melhor extrapolação e validação dos dados, entretanto, deve-se ter 0 
cuidado de não seguir o fluxo oposto e desprezar a população masculina, fato que também reduziria a criação de conteúdos de qualidade (HOLDCROFT A, 2007). Embora tais fatos constituam uma justificativa ética e integral, segundo os critérios do National Institutes of Health (2019) para a realização de ensaios clínicos, visto que já existe uma comparação entre os sexos que corrobora que ser do sexo feminino constitui fator de risco.

Vale ressaltar que proteger o sexo feminino se torna uma estratégia para redução da doença mental no futuro dela e de outros adultos. Por exemplo, cuidar da saúde da gestante, pode prevenir que após o parto ela se torne uma mãe depressiva, estas, quando em contato com suas crianças podem predispô-las a depressão no futuro, constituindo o fator biopsicossocial da doença (WAINBERG ML, et al., 2017).

Comparativamente, o percentual de crianças admitidas em estudos relacionados à depressão foi inferior ao de outras populações, o que não é demonstrado em estudos sobre ansiedade. Isso pode estar relacionado ao fato apontado por Egger HL e Angold A (2006), uma vez que indivíduos menores de 17 anos apresentam maior número de transtornos de ansiedade do que depressão, enquanto em adultos ocorre o contrário.

O estímulo a pesquisa de saúde mental em crianças e com intervenções adequadas neste momento da vida é uma área promissora, uma vez que nesta faixa etária os diagnósticos podem ser subestimados e o tratamento feito de forma incorreta, corrigir estes erros pode ser uma forma de prevenção debilidades e doenças em adultos (WAINBERG ML, et al., 2017).

Os idosos tiveram grande participação nos estudos sobre depressão, sendo ainda a que mais contribuiu para a divulgação dos estudos, com $14,03 \%$ deles com resultados. Isso pode ser justificado pela alta prevalência de depressão nessa população, que pode variar de 5 a $8 \%$ em ambos os sexos, e também pelo aumento, com o passar da idade, da incidência de depressão maior (OMS, 2017; GHDX, 2017).

Assim, percebe-se para esta patologia um fenômeno diferente da sub-representação ocorrida anteriormente, a qual limitava as informações sobre eficácia, segurança e a extrapolação dos dados para essa amostra (SHENOY P e HARUGERI A, 2015; HERRERA AP, et al., 2010). Os benefícios que podem se observar com isso são equidade e justiça para essa população em questão de saúde, generalização dos resultados com número ideal de participantes, compreender efeitos adversos em idosos e interações com outras drogas de uso contínuo e geral, além das necessidades de saúde dos mesmos (SHENOY P e HARUGERI A, 2015).

Por fim, os transtornos mentais comuns possuem grande espaço na pesquisa científica se comparados a transtornos mais severos, como os psicóticos, embora estes possam ser mais fáceis de diagnosticar com critérios objetivos. Isso culmina numa queda de tratamentos eficazes de indivíduos doentes e pouca informação a cerca desse tema (WAINBERG ML, et al., 2017).

Entretanto ainda é preciso, para as primeiras, considerar melhores análises e transparência para que a conduta seja embasada em evidências fidedignas. Neste ponto, boa parte dos estudos podiam estar relacionados a medicação, de acordo com a maior fonte de financiamento ser a indústria, mas a prática médica não deve ser obscurecida por este fato e outras terapêuticas, como terapias comportamentais e psicanalítica já validadas devem ser prescritas (WAINBERG ML, et al., 2017).

\section{CONSIDERAÇÕES FINAIS}

A ansiedade torna-se cada vez mais prevalente em diferentes populações, embora ainda seja negligenciada até pelos próprios pacientes, visto que a linha entre patologia e normalidade é tênue para esta, exigindo melhores critérios diagnósticos. É cada vez mais importante pensar em vieses, não só para os ensaios clínicos, valorizando a ética na publicação de resultados positivos e negativos em todas as circunstâncias observadas, para evitar que os danos se espalhem para pacientes e participantes do estudo no futuro, tomando o cuidado de garantir a equidade para todas as populações estudadas. A prática médica deve fornecer tudo ao seu alcance para a melhoria dos pacientes, sendo farmacológico e não farmacológico conhecendo as limitações de ambos. Por fim, outras áreas da saúde mental também merecem mais atenção da pesquisa científica para produção de dados verossímeis. 


\section{REFERÊNCIAS}

1. ALLIED MARKET RESEARCH. Antidepressant Drugs Market Expected to Reach \$15,983 Million by 2023.2018. Disponível em: < https://www.alliedmarketresearch.com/press-release/antidepressants-drugs-market.html>.

2. CLINICAL TRIALS. Clinical Trials.gov. 2019. Disponível em: < https://www.clinicaltrials.gov/>.

3. DOSHI P, et al. Restoring invisible and abandoned trials: a call for people to publish the findings. Bmj, $2013 ; 346$ (132):2865-2865.

4. EGGER HL, ANGOLD A. Common emotional and behavioral disorders in preschool children: presentation, nosology, and epidemiology. Journal of Child Psychology and Psychiatry, 2006; 47(3-4): 313-337.

5. FELDMAN AM. Publishing "Invisible" and "Abandoned" Clinical Trials: a commitment for cts. Clinical and Translational Science, 2013; 6(4): 251-253.

6. GHDx. Global Health Data Exchange. GBD Results Tool about Death and Disability- Adjusted years of life $1990-2017$. 2017. Disponível em: <http://ghdx.healthdata.org/gbd-results-tool>.

7. HEGEMAN JM et al. Phenomenology of depression in older compared with younger adults: meta-analysis. Brazilian Journal of Psychiatry, 2012; 200:275-81.

8. HELP GUIDE. Anxiety Medication. 2020. Disponível em: <https://www.helpguide.org/articles/anxiety/anxietymedication.htm>.

9. HERRERA AP, et al. Disparate Inclusion of Older Adults in Clinical Trials: priorities and opportunities for policy and practice change. American Journal of Public Health, 2010; 100 (1): 105-112.

10. HOLDCROFT A. Gender bias in research: how does it affect evidence based medicine? Journal of the Royal Society of Medicine, 2007 Jan; 100(1): 2-3.

11. HWANG TJ, et al. Failure of Investigational Drugs in Late-Stage Clinical Development and Publication of Trial Results. JAMA Internal Medicine, 2016;176(12):1826-1833.

12. KUNSEL T, SUMANT O. Antidepressant Drugs Market by Depressive Disorder (Major Depressive Disorder, Obsessive-Compulsive Disorder, Generalized Anxiety Disorder, Panic Disorder, and Others) and Product (Tricyclic Antidepressants, Selective Serotonin Reuptake Inhibitors, Serotonin-Norepinephrine Reuptake Inhibitors, Monoamine Oxidase Inhibitors, Serotonin Antagonist and Reuptake Inhibitors, and Others) - Global Opportunity Analysis and Industry Forecast, 2017-2023. Allied Market Research (Online), 2018.

13. LISKAUSKAS S, et al. Changing times for science and the public: Science journalists' roles for the responsible communication of science. EMBO reports, 2019; 20(4): 1-4.

14. MARI JJ, OQUENDO MA. Mental health consequences of COVID-19: the next global pandemic. Trends In Psychiatry And Psychotherapy, 2020, 42 (3): 219-220.

15. MURCHO N, et al. Transtornos mentais comuns nos Cuidados de Saúde Primários: Um estudo de revisão. Revista Portuguesa de Enfermagem de Saúde Mental, 2016; (15):30-36.

16. NIH. National Institutes of Health. Enhancing Reproducibility through Rigor and Transparency. Grants and Funding (Online), 2018.

17. NIH. National Institutes of Health. Guidelines for the Review of Inclusion on the Basis of Sex/Gender, Race, Ethnicity, and Age in Clinical Research, 2019: 8 p.

18. PÁDUA GCC, GUILHEM D. Integridade científica e pesquisa em saúde no Brasil: revisão da literatura. Revista de bioética, 2015; 23 (1): 124-38.

19. PINTO AC. Integridade Científica: Compromisso Da SBQ. Quím. Nova. 2015; 38(3): 297.

20. POLLITT A, et al. Project Ecosystem: mapping the global mental health research funding system. Reino Unido: Rand Europe, 2016. 69 p.

21. RIVEROS C, et al. Timing and Completeness of Trial Results Posted at ClinicalTrials.gov and Published in Journals. Plos Medicine, 2013; 10(12): 1-12.

22. SHENOY P, HARUGERI A. Elderly patients' participation in clinical trials. Perspectives In Clinical Research, 2015; 6(4):184-189.

23. SMITH MA, et al. (Reviewer). Anxiety Medication [Online]. Help Guide; 2020.

24. VASCONCELOS S, et al. Brazilian Science and Research Integrity: Where are We? What Next? Anais Acadêmicos Brasileiros de Ciências, 2015; 87(2):1259-69.

25. WAINBERG ML, et al. Challenges and Opportunities in Global Mental Health: a research-to-practice perspective. Current Psychiatry Reports, 2017; 19 (5): 28-44.

26. WHO. World Health Organization. COVID-19 disrupting mental health services in most countries, WHO survey, 2020. Disponível em: <https://www.who.int/news/item/05-10-2020-covid-19-disrupting-mental-health-services-in-mostcountries-who-survey>. 